\title{
eHealth Literacy Among Health Sciences Students in Greece
}

\author{
Trisevgeni TRANTALI ${ }^{\mathrm{a}}$, Christina ATHANASOPOULOU ${ }^{\mathrm{b}}$, Areti LAGIOU $^{\mathrm{c}, \mathrm{d}}$ and \\ Evanthia SAKELLARI ${ }^{\mathrm{c}, \mathrm{d}, 1}$ \\ a Master's Programme "Health Promotion and Health Education", National and \\ Kapodistrian University of Athens \& University of West Attica, Athens, Greece \\ ${ }^{\mathrm{b}}$ Department of Occupational Therapy, School of Health and Care Sciences, University \\ of West Attica, Athens, Greece \\ ${ }^{c}$ Department of Public and Community Health, School of Public Health, University of \\ West Attica, Athens, Greece \\ ${ }^{d}$ Laboratory of Hygiene and Epidemiology, University of West Attica, Athens, Greece
}

\begin{abstract}
Health literacy is a necessary skill to find and make good use of online health information. However, the general public lacks this skill; it is essential for future health professionals to be able to guide and facilitate the public. The study aimed to examine the perceived eHealth literacy level of health sciences students in Greece. A cross-sectional, online survey was conducted ( $\mathrm{N}=113$ students). The questionnaire included socio-demographic data and the eHealth Literacy Scale (eHEALS). Participants' mean eHEALS score was 31.9 with medicine and dentistry students having the highest score (33.7) and other health and caring sciences students the lowest (29.8). There was no statistically significant difference at eHEALS score among participants of different academic year. However, there was a statistically significant difference at eHEALS score among University Departments $(\mathrm{p}=0.009)$. Further research in representative samples is required to assess specific needs and improve current educational curricula.
\end{abstract}

Keywords. eHealth literacy, eHEALS, health information, health sciences students

\section{Introduction}

The internet is a major source of health information [1]. So, "the ability to find, understand, and apply online health information in order to make appropriate health decisions", known as eHealth literacy, is a necessary skill [2]. However, previous studies have shown that not only the general public lacks of this skill [3] but also the health professionals [4]. Investigating eHealth literacy among future health professionals plays an important role as they help in guiding and facilitating the general public to find, understand and apply online health information. Especially in Greece, there is limited literature about eHealth literacy among health sciences students. Thus,

\footnotetext{
${ }^{1}$ Corresponding Author, Evanthia Sakellari, Department of Public and Community Health, Laboratory of Hygiene and Epidemiology, School of Public Health, University of West Attica, 196 Alexandras Av., 11521, Athens, Greece; E-mail: sakellari@uniwa.gr.
} 
the aim of this study was to examine the perceived eHealth literacy level among undergraduate health sciences students in Greece.

\section{Methods}

A cross-sectional, online survey was conducted between June $4^{\text {th }}$ and July $13^{\text {th }}, 2021$. A convenient sample of health sciences students from Greek Universities was recruited using a passive recruitment strategy, through invitation via Facebook groups. The study was granted permission from the Ethics Committee of the University of West Attica (41141-25/05/2021).

The eligibility criteria were: 1) internet access 2) 18 years of age or older, 3) undergraduate health sciences student at Greek University 4) understanding Greek language 5) accepting to complete the questionnaire voluntarily and anonymously.

Data collection was made using a structured, self-administered questionnaire through Google Forms, which consisted of 16 items: 1) socio-demographic characteristics (8 items), 2) eHealth Literacy Scale-eHEALS (8 items) [5,6], measured participants' combined knowledge, confidence and perceived skills in finding, evaluating and applying eHealth information to health problems. Each item was scored on a 5-point Likert scale, from 1 "Strongly disagree" to 5 "Strongly agree" (min:8; max:40). Higher score represented higher perceived eHealth literacy level. In this study the eHEALS had an internal consistency of Cronbach $\mathrm{a}=0.89$.

Data analysis was made using SPSS Version 26. Descriptive statistics were used to identify participants' socio-demographic characteristics and eHealth literacy scores. Moreover, One way Analysis of Variance on ranks (Kruskal-Wallis Test) was used to compare the eHEALS scores among participants. The statistical significance level was set at 0.05 [7].

\section{Results}

The total study sample was 113 . Median age was 22 years, the majority were female $(n=92,81.4 \%)$, single $(n=65,57.5 \%)$, students at universities of Attica region $(n=90$, $79.6 \%)$, at $2^{\text {nd }}$ academic year $(n=34,30 \%)$, who studied health and caring sciences (midwifery, occupational therapy, physiotherapy or nursing) $(\mathrm{n}=37,32.7 \%)$ (Table 1).

Table 1. Socio-demographic characteristics of participants.

\begin{tabular}{lc}
\hline \multicolumn{1}{c}{ Socio-demographic characteristics } & Total sample $\mathbf{N}=\mathbf{1 1 3}$ \\
Age: Median (min, max) & $22(18,53)$ \\
Gender: N (\%) & $19(16.8)$ \\
Male & $92(81.4)$ \\
Female & $2(1.8)$ \\
Other & \\
Marital status: N (\%) & $65(57.5)$ \\
Single & $42(37.2)$ \\
Partnership/Married & $6(5.3)$ \\
Other & \\
University: N (\%) & $90(79.6)$ \\
Attica region & $23(20.4)$ \\
Outside Attica region &
\end{tabular}




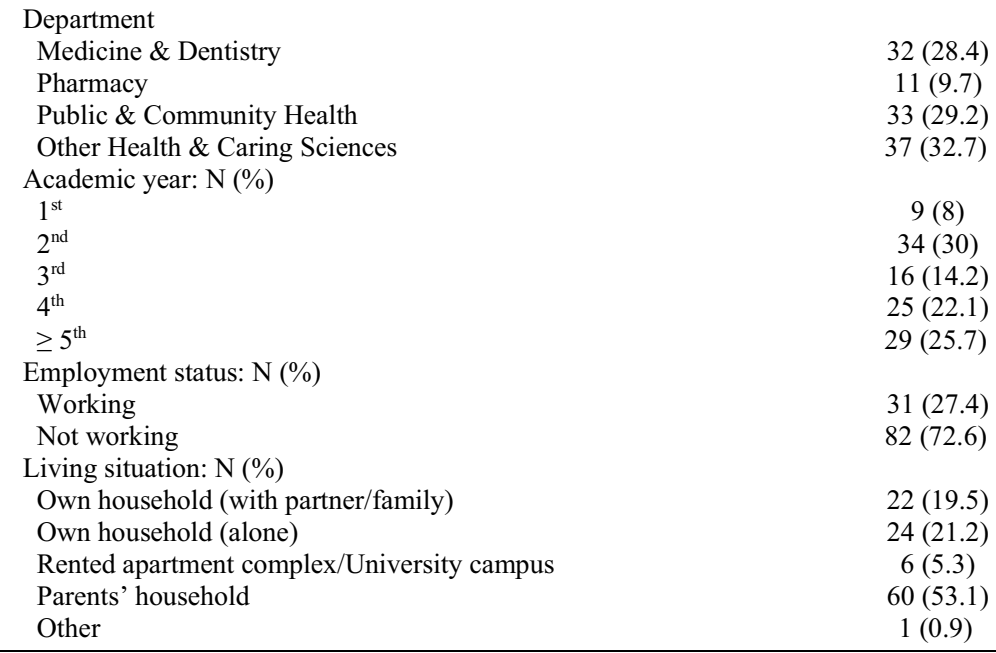

The mean total eHEALS score was 31.9 (SD: 4.9, min: 18; max: 40). Table 2 below, shows the mean scores of individual items of the eHEALS scale (min:1; max:5). The lowest scoring item was 3.4, indicating the weakest eHealth literacy item while the highest scoring item was 4.2 , indicating the strongest eHealth literacy item.

Table 2. Participants' score (Mean \& SD) on the eHEALS items.

\begin{tabular}{|c|c|}
\hline eHEALS Items & Mean (SD) \\
\hline I know what health resources are available on the Internet & $3.9(0.7)$ \\
\hline I know where to find helpful health resources on the Internet & $4.1(0.8)$ \\
\hline I know how to find helpful health resources on the Internet & $4.2(0.7)$ \\
\hline I know how to use the Internet to answer my questions about health & $4(0.8)$ \\
\hline I know how to use the health information I find on the Internet to help me & $4(0.8)$ \\
\hline I have the skills I need to evaluate the health resources I find on the Internet & $4.1(0.7)$ \\
\hline I can tell high-quality health resources from low-quality health resources on the Interne & $4.1(0.8)$ \\
\hline I feel confident in using information from the Internet to make health decisions & $3.4(1.1)$ \\
\hline
\end{tabular}

No significant difference in terms of total eHEALS scores was observed among participants of different academic years. However, there was a statistically significant difference of total eHEALS score among the different departments represented in the study (Table 3). Further statistical analysis using Bonferroni correction indicated that the mean eHEALS score of medicine and dentistry students (mean: 33.7) was higher than the score of other health and caring sciences students (mean: 29.8) (Bonferroni Post Hoc Test, $\mathrm{p}=0.004$ ).

Table 3. Comparison of the total eHEALS scores between departments and academic years.

\begin{tabular}{llcccc}
\hline & \multicolumn{1}{c}{ Variable } & \multicolumn{3}{c}{ Total eHEALS score } & \\
& Mean & Median & Min, Max & p-value* \\
\hline \multirow{2}{*}{ Department } & Medicine \& Dentistry & 33.7 & 33.5 & 23,40 & \\
& Pharmacy & 31.8 & 31 & 26,40 & 0.009 \\
& Public \& Community Health & 32.6 & 32 & 22,40 & \\
& Other Health \& Caring Sciences & 29.8 & 30 & 18,40 & \\
Academic & $1^{\text {st }}$ & 31.3 & 32 & 18,40 & \\
& $2^{\text {nd }}$ & 30.3 & 31 & 21,40
\end{tabular}




\begin{tabular}{lllll} 
year & $3^{\text {rd }}$ & 32.7 & 32 & 26,40 \\
& $4^{\text {th }}$ & 32.9 & 32 & 22,40 \\
& $\geq 5^{\text {th }}$ & 32.8 & 32 & 27,40 \\
\hline
\end{tabular}

* Kruskal-Wallis rank test

\section{Discussion}

This is the first study examining the perceived eHealth literacy among health sciences students in Greece. The current study found a higher mean eHEALS score (31.9) among health sciences students compared to the score found in previous similar studies examining eHEALS score among medical and health sciences students (mean: 28.21) [8] and nursing students (mean: 24.52) [9]. Due to the small number of participants, the study results cannot be generalized. Further research is needed in order to assess the characteristics and specific needs. Moreover, the examination of additional information such as more socio-economic characteristics, internet usage and devices, primary connectivity in relation to eHEALS score among health sciences students could provide more specific data in this field. In conclusion, based on this important insight, departments could develop curricula that emphasize on practical eHealth literacy skills and critical thinking, in order to educate the future health sciences professionals.

\section{References}

[1] Eurostat. One in two EU citizens look for health information online. 2021. Available at: https://ec.europa.eu/eurostat/web/products-eurostat-news/-/edn-20210406-1.

[2] Norman CD, Skinner HA. eHealth Literacy: Essential Skills for Consumer Health in a Networked World. J Med Internet Res. 2006 June;8(2):e9.

[3] Park H, Cormier E, Gordon G, Baeg JA. Identifying Health Consumers' eHealth Literacy to Decrease Disparities in Accessing eHealth Information. Comput Inform Nurs. 2016 Feb;34(2).

[4] Stellefson M, Hanik B, Chaney B, Chaney D, Tennant B, Chavarria EA. eHealth Literacy Among College Students: A Systematic Review With Implications for eHealth Education. J Med Internet Res. 2011 Dec;13(4):e102.

[5] Norman CD, Skinner HA. eHEALS: The eHealth Literacy Scale. J Med Internet Res. 2006 Nov;8(4):e27.

[6] Athanasopoulou C, Valimaki M, Koutra K, Lottyniemi E, Bertsias A, Basta M, Vgontza A, Lionis C. Internet use, eHealth literacy and attitudes toward computer/internet among people with schizophrenia spectrum disorders: a cross-sectional study in two distant European regions. BMC Med Inform Decis Mak. 2017 Sept;17(123).

[7] Crosby R, DiClemente R, Salazar L. Research methods in health promotion. San Francisco: JosseyBass; 2006. 317-346 p.

[8] Dashti S, Peyman N, Tajfard M, Esmaeeli H. E-Health literacy of medical and health sciences university students in Mashhad, Iran in 2016: a pilot study. Electron Physician. 2017 Mar; 9(3):39663973.

[9] Tanaka J, Kuroda H, Igawa N, Sakurai T, Ohnishi M. Perceived eHealth Literacy and Learning Experiences Among Japanese Undergraduate Nursing Students: A Cross-sectional Study. Comput Inform Nurs. 2020 Jan-Mar;4(1):e2. 